\title{
Changes in attentional processing and affective reactivity in pregnancy and postpartum
}

\author{
This article was published in the following Dove Press journal: \\ Neuroscience and Neuroeconomics \\ II November 2014 \\ Number of times this article has been viewed
}

\section{Jackie K Gollan \\ Laina Rosebrock \\ Denada Hoxha \\ Katherine L Wisner}

Asher Center for the Study and Treatment of Depressive Disorders, Department of Psychiatry and Behavioral Sciences, Northwestern University Feinberg School of Medicine, Chicago, IL, USA
Correspondence: Jackie K Gollan Asher Center for Study and Treatment of Depressive Disorders, 676 North St Clair Street, Chicago, IL 606I3, USA

Tel + I 3|2 $695612 \mid$

$\mathrm{Fax}+\mathrm{I} 3126955502$

Email j-gollan@northwestern.edu
Abstract: The aim of this review is to provide an overview of the research in attentional processing and affective reactivity in pregnancy and postpartum to inform future research. Numerous changes occur in attentional processing and affective reactivity across the childbearing period. This review focuses on the definition and methods of measuring attentional processing and affective reactivity. We discuss research studies that have examined the changes in these two processes during the perinatal phases of pregnancy and postpartum, with and without depression and anxiety. We evaluate the importance of using multiple levels of measurement, including physiological and neuroimaging techniques, to study these processes via implicit and explicit tasks. Research that has identified regions of brain activation using functional magnetic resonance imaging as well as other physiological assessments is integrated into the discussion. The importance of using sophisticated methodological techniques in future studies, such as multiple mediation models, for the purpose of elucidating mechanisms of change during these processes in pregnancy and postpartum is emphasized. We conclude with a discussion of the effect of these processes on maternal psychological functioning and infant outcomes. These processes support a strategy for individualizing treatment for pregnant and postpartum women suffering from depression and anxiety.

Keywords: attentional processing, emotion, affective reactivity, depression, pregnancy, postpartum

\section{Introduction}

Research has examined the emotional, cognitive, and biological changes that occur during pregnancy and postpartum to increase our understanding of the physiology of transition to motherhood and its effect on maternal and infant well-being. During the postpartum period, women are more likely to experience anxiety, sleep irregularities, increased neuroticism, and other depressive symptoms relative to nonpartum women. ${ }^{1,2}$ These emotional disturbances have associated neural correlates, both in the context of general affective response and regulation as well as when responding to infant cues (for a review, see Pechtel and colleagues ${ }^{3}$ ). Dysregulation of the mother's emotional state and correlated neural responses, particularly in affective and cognitive control centers such as the amygdala and prefrontal cortex, may impair attachment with her infant. Although the literature has been consistent in showing that emotional disturbances are common throughout this critical period and exert negative effects on attachment, specific mechanisms responsible for these changes are not well understood. In addition, the pathophysiology of the development and maintenance of depressive and other clinical symptoms during pregnancy and postpartum remain understudied. 
Data suggest that cognitive reorganization occurs during pregnancy, ${ }^{4}$ associated with minor impairments in working memory, free recall, and general cognition, and specifically the domains of processing speed and abstract reasoning. These changes have implications for the way women process and respond to affective information in their environment and how they may organize their behaviors in response to stimuli unique to motherhood or mother-baby interactions. Differences in cognitive processing have also been found in the perception of cognitive abilities during pregnancy. During the third trimester, pregnant women have reported impairments in attention and memory, although performance on objective measures remained intact. ${ }^{5}$ Perception of impairments in these areas may lead to important behavior changes, particularly if the mother perceives she is poorly equipped to handle the demands of motherhood.

Attention and affective changes may be adaptive and provide protection for both mother and infant against potential environmental and biological threats. For example, a heightened response to threat ${ }^{6}$ allows for a faster and more targeted response to protect the infant from external harmful stimuli. Pregnant and postpartum women have shown cognitive enhancements in certain tasks and outperform nonpartum controls. An example is a "stem completion and recognition" task. Pregnant and nonpregnant women were both asked to recall previously presented words prompted only with ambiguous letters. Pregnant women remembered significantly more words related to motherhood compared with nonpregnant women. ${ }^{7}$ The greater attunement to pregnancy and motherhood-related stimuli may be evolutionarily adaptive as women transition to caring for an infant. However, this research remains limited, particularly in the domain of specific implicit and explicit cognitive processes such as attention and affective reactivity. Given the potential effect on mother-infant relationships and infant outcomes, this is an area that compels further study.

Biological changes appear to have a strong influence on these cognitive and affective disturbances. Many neurobiological and endocrine changes occur during fetal development; including increases in sex steroid hormone secretion (ie, prolactin and oxytocin), pregnancy-specific peptide hormone secretion, and expression of specific hormone receptors in the brain. ${ }^{8}$ These changes have pervasive influences on behavioral and emotional expression in mothers, including lactation, ovulation suppression, stress response reduction, and appetite increase. In particular, hormone levels (eg, oxytocin) play a role in mood changes and reduced performance on mental tasks and amnesic effects. ${ }^{9,10}$ In addition, neuropeptide expression in neurons during pregnancy has an effect on stress responses, such that pregnant women show a decreased stress response via the hypothalamicpituitary-adrenal axis; however, responses still exist, and women who demonstrate increased chronic - not brief acute - stress during pregnancy are more likely to have suboptimal outcomes such as preterm delivery. ${ }^{11}$ Furthermore, research has linked individual differences in maternal attachment to the development of dopaminergic and oxytocinergic systems, showing that the oxytocin response to both infant contact and viewing their own infant's faces was increased in women with secure attachment. ${ }^{12}$

Taken together, this research indicates that changes in affective, cognitive, and behavioral responses to one's environment are key features of the perinatal phase. These alterations may appear as a result of the vast neurobiological changes that occur to prepare a woman's body for parturition and motherhood. For a more detailed and extensive review on this literature, we refer the interested reader to a special issue of Progress in Brain Research focused on brain activation and other biological changes and on their associations with maternal functioning. ${ }^{8}$

This review will focus on two specific processes during the perinatal phase, attentional processing and affective reactivity, which occur outside of awareness and yet have a significant influence on behavior, learning, decision making, and emotional experience. Attentional processing has particular salience during the peripartum transition, as mothers must become increasingly aware and vigilant of their environments to protect themselves and their infants from incoming threats. Disruption of this process has implications for a mother's capacity to respond well to her infant. In addition, affective reactivity, which reflects a mother's sensitivity and immediate response to emotional stimuli, has a direct effect on how a mother responds to her environment, particularly toward signals from her infant. ${ }^{3}$ We summarize and discuss the existing research on attentional processing and affective reactivity in pregnant and postpartum women across different assessment modalities and measurement techniques. Similarities in these processes between perinatal and nonperinatal samples will be discussed. The influence of depression on these processes and potential application to treatment provides a direction for future research and methodological techniques. In addition, the effect any disruptions in these processes have on infant outcomes is considered in each section.

\section{Attentional processing in pregnancy and postpartum}

Attentional processing refers to the cognitive processing that determines which external information is attended to, 
and consequently involves implicit cognitive processes that influence behavior. ${ }^{13-15}$ Negative attentional bias is characterized by preferential attention to negative stimuli over neutral stimuli when the two stimuli sets compete in an implicit experimental task for processing priority. ${ }^{16}$ Functionally, attentional bias represents an individual's difficulty shifting attention away from negative stimuli, such that goal-irrelevant affective stimuli unduly influence cognitive processing and subsequent behavior. Attentional biases involving affect have been studied with standardized emotional imagery, including emotional faces and emotional environmental scenes. ${ }^{17,18}$ Research has indicated that attention is oriented away from sad faces and toward happy faces in healthy subjects ${ }^{19}$ or is distributed evenly across valenced and neutral faces. ${ }^{20}$ This may be a function of survival relevance, as opposed to valence, as attention is preferentially engaged by threat signals such as fearful faces, ${ }^{21,22}$ and neutral infant faces versus neutral adult faces, ${ }^{23}$ thus ensuring adequate resources are allocated for protection from harm and survival of offspring, respectively.

Attentional bias can be influenced by an individual's mood. For example, depression is characterized by greater attentional bias to negative stimuli. . $^{1920,24,25}$ Individuals with depressed mood allocate attentional resources to sad stimuli over happy or neutral ones. Notably, attentional bias plays a causal role in the maintenance of depression, ${ }^{26}$ and when attentional bias decreases, depression symptoms remit ${ }^{27}$ and stress recovery can occur. ${ }^{28}$ Anxiety has been characterized by overly heightened bias to threat cues ${ }^{29,30}$ and preferential eye gaze toward intense negative facial expressions, particularly for increased anxious symptomatology. ${ }^{31}$ This may perpetuate the heightened reactivity to seemingly innocuous stimuli characteristic of pervasive and specific anxiety. Finally, attentional bias to threat-related stimuli has been shown to be causally related to the development of anxiety, ${ }^{32}$ although its causal role in perinatal depression is unclear.

Attentional bias is characterized by deficits in nondepressed women with high-risk pregnancies compared with women with uncomplicated pregnancies. ${ }^{33}$ Implicit attention toward infant distress has been associated with improved maternal-infant interactions in nondepressed pregnant women ${ }^{14,34}$ and secure attachment development in infants. ${ }^{35}$ Infant faces may be more salient to mothers, as evidenced by longer response to times to infant versus adult faces in healthy mothers compared with nonmothers. ${ }^{36}$ Increased attention and responsiveness to infants and infant distress is evolutionarily adaptive and ensures a mother attends to her infant's needs. Conversely, depressed pregnant women have shown significantly decreased attentional bias for infant distressed faces compared with healthy pregnant women. ${ }^{37}$ This effect was not found for adult faces. Nondepressed women were slower to disengage from distressed infant faces, whereas depressed women showed the opposite trend and were quicker to disengage from these faces. In addition, antenatal factors such as attachment style and self-identity influence preferential processing and maternal sensitivity. ${ }^{14,38,39}$

These irregularities in preferential processing toward distressed infant cues suggest depressed mothers may be less likely to attend to incoming threats. Modulation of the startle reflex, an implicit measure of threat response and defense system mobilization, is attenuated among healthy women between 4-6 weeks postdelivery, which suggests a "deactivated defense system." 40 Although no studies have specifically looked at startle modulation in depressed women during pregnancy or postpartum, it is possible this response is further attenuated because of the blunted reactivity common in depression..$^{41}$ Review of neuroimaging data indicates that healthy mothers have brain activation in reward centers when explicitly attending to their own infant's face relative to unknown infants, ${ }^{42-46}$ which is proposed to keep a mother engaged with the stimulus and her infant. In mothers with insecure attachment styles, these same reward processing networks were not activated in response to distressed faces ${ }^{43}$ and were associated with insecure attachment. ${ }^{12}$ In addition, mothers who reported higher maternal care showed increased activation in areas associated with greater emotional processing and representation of others' mental states. ${ }^{47}$

In summary, research in attentional bias in nonpartum depressed samples is available, whereas research on attentional bias in pregnancy and postpartum samples is scant. However, we can glean the following observations from the existing data: First, healthy pregnant and postpartum women show a heightened attunement of implicit attention to threat and infant signals relative to nonperinatal women. Second, attentional indices to threat and infant stimuli have distinct neurocognitive and physiological correlates. Third, attentional processing appears to be influenced by other trait factors, specifically attachment style, as well as state factors, such as mood and the presence of depression or anxiety symptoms. Fourth, this altered attentional processing has implications for mother-infant interactions by decreasing a mother's implicit response to her infant's distress. New research may seek to clarify the neurocognitive and physiological mechanisms of attentional processes 
toward identifying targets for treatment for perinatal samples.

\section{Affective reactivity in pregnancy and postpartum}

Affective reactivity is characterized by an individual's detection and responsivity to emotional stimuli. Affect, or subjectively experienced feelings, is central to mood and emotions, is often expressed in a preconscious way (without the person explicitly describing their emotions, and therefore requiring testing using computer-generated tasks that elicit affective reactions), and may be measured using two dimensions. The first dimension is valence, which specifies feelings from neutral-pleasant (positivity) and neutral-unpleasant (negativity) evoked by emotional information. ${ }^{48}$ The second dimension is arousal, defined as the intensity of response to the stimuli. ${ }^{48,49}$ Using standardized, well-validated tasks that use emotional stimuli, such as the International Affective Picture System, ${ }^{18}$ or facial expressions depicting emotions, ${ }^{17}$ the person can assign ratings of valence and arousal to reflect their affective reactivity.

Affective reactivity has been measured in both healthy and clinical participants, with important differences. In general, healthy participants rate highly negative and positive information as more arousing than neutral information. ${ }^{48}$ Conversely, depressed participants have attenuated ratings to both positive and negative images, ${ }^{41}$ indicating a blunted reactivity that may ultimately influence approach and avoidance behavior. In terms of facial expressions, depression has been associated with interpretations of ambiguous or neutral facial expressions as negative ${ }^{50}$ and difficulty detecting happiness in mildly pleasant facial expressions. ${ }^{51}$ Differentially, anxious participants have shown heightened arousal ratings to emotional stimuli, specifically threatening or pathologyspecific fear cues. ${ }^{52,53}$ These findings suggest an underlying difference in attention and affective responding to affective cues in psychopathology. It is unclear whether this is causative or an effect of depression/anxiety, although research indicates it may be a vulnerability factor for the development of emotional distress. ${ }^{54,55}$

Functionally, mothers are continually prompted to interpret and react to the environment, and particularly cues from their babies, with emotional responses that ensure infant- and self-care. Systematic biases in emotional reactions, characterized by distorted reactivity to emotional information, have critical relevance to decision-making, cognition, and health and, by extension, are likely to have important effects on maternal affective experience. Distorted affective reactivity may affect mother-infant interactions, quality of attachment, and the infant's affective experiences. ${ }^{56}$ The presence of depressive symptoms in mothers, which has been shown to influence affective reactivity, has negative consequences for child cognitive development and behavior. ${ }^{57}$ This may have an effect on implicit affective processing in offspring as well. For example, daughters of depressed mothers selectively attended to negative facial expressions, whereas daughters of nondepressed mothers attended more to positive facial expressions. ${ }^{58}$

Depressed postpartum women have shown a negative interpretation of neutral infant and adult faces, ${ }^{59,60}$ poorer recognition of negative stimuli compared with nonpartum depressed women, ${ }^{61}$ and decreased accuracy in identifying happy faces. ${ }^{62}$ This indicates a disruption in the recognition and processing of emotional stimuli, as indicated by explicit responses to these stimuli, which reflects underlying implicit processes. Difficulties in discerning infant-related affective cues may have deleterious effects on mother-baby dyads and attachment processes. Research on affective reactivity in pregnancy is scarce, but one study demonstrated that pregnant women with depression show attenuated intensity to emotional stimuli compared with pregnant women without depression. ${ }^{63}$ Taken together, these findings indicate that pregnant and postpartum women have altered implicit emotional information processing, and therefore explicit emotional response. However, future research should compare healthy pregnant and postpartum women with age-matched nonpartum women to delineate whether this is a result of clinical symptoms, pregnancy, or both. In addition, research should focus on better understanding the effect of affective reactivity processes on mother-baby attachment processes in both clinical and nonclinical samples, using longitudinal methodology.

Neuroimaging research has indicated that healthy pregnant women in their second trimester show increased activation to fear-related stimuli in the prefrontal cortex compared with those in the third trimester. In addition, in this study, activation of the prefrontal cortex while processing fearful faces was negatively associated with selective attention to fearful faces and was positively associated with general distress and anxiety. ${ }^{64}$ Overall, this suggests an alteration of prefrontal cortex function during the processing of fear-relevant stimuli in pregnancy, which may occur as a result of psychobiological changes during this trimester and may be a vulnerability for depression and anxiety during postpartum. However, neuroimaging studies in human pregnancy are scarce, with most research being performed 
on rats and mice. ${ }^{65}$ Future research may investigate this topic with humans.

Parents in general, and mothers in particular, also demonstrate neural activation differences compared with nonparents, such as deactivation in the anterior cingulate cortex and a stronger reaction to a crying than a laughing infant. ${ }^{66}$ First-time healthy mothers demonstrate activation of an extensive brain network when viewing pictures of their own infant, including the ventral tegmental area/substantia nigra, the striatum, and the frontal lobe regions involved in emotion, cognition, and motor/behavioral outputs. ${ }^{43,67}$ In contrast, women with PPD have lower activation in the posterior orbitofrontal cortex for negative versus neutral stimuli ${ }^{68}$ and decreased activation in the left dorsomedial prefrontal cortex and amygdala while viewing infant faces. ${ }^{69}$ Ultimately, disruption in reactivity to emotional information may activate different brain networks and influence subsequent behavior, which affects functioning and impairs mother-infant interactions. Understanding these underlying brain networks may provide crucial information about neural substrates or vulnerabilities and illuminate treatment approaches to help mothers engage optimally with their infants.

In summary, the research on affective reactivity in perinatal populations supports several conclusions. First, there are specific brain networks that activate in healthy pregnant and postpartum women when viewing pictures of their own infants; in particular, reward-related brain regions are crucial for increasing motivation to engage in infant caregiving. Heightened activation to threat stimuli occurs during pregnancy in preparation for motherhood and continues in parenthood. Second, depressed mothers show altered affective reactivity in the form of decreased brain activation in areas, such as the amygdala and prefrontal cortex, with attenuated arousal, but potentiated negative ratings in response to emotional stimuli and impaired recognition of emotional faces. Third, these biases have implications for mother-infant attachment and even implicit processes in offspring. However, the direct influences of affective reactivity, as well as potential targets for treatment, remain less understood and provide an avenue for future research.

\section{Necessity for sample-specific and sample-validated stimuli}

Within the realm of research in attentional processing and affective reactivity, several standardized emotional stimuli sets are used in experimental paradigms. When studying samples of pregnant and postpartum women, many researchers have created study-specific stimuli such as images of the mother's own infants or filming adults and infants from the surrounding community in naturalistic settings; this is because a standardized set of maternal-specific stimuli is not currently available. ${ }^{37,43,59,70}$ Scientific testing of these stimuli sets requires time, effort, and resources.

The validation process for standardized stimuli sets such as the International Affective Picture System, ${ }^{18}$ as well as study-specific images, includes presentation of these stimuli to college students, research assistants, and convenience samples from the community. ${ }^{59}$ This is crucial for ensuring that stimuli are truly representative of what they are meant to convey (a sad face is consistently viewed as sad by norms composed of nonclinical pregnant and postpartum women). However, different populations, such as clinical samples or pregnant/postpartum women, might view stimuli differently than healthy individuals. In a study examining affective reactivity in pregnant and postpartum women while viewing International Affective Picture System images, arousal ratings of each picture were compared with normative ratings of the same stimuli by female college students. Results showed that healthy pregnant and postpartum women assigned lower arousal ratings to neutral, positive, and threatening images compared with healthy college women. These observations indicate a blunting of reactivity in healthy women during pregnancy and postpartum..$^{63,71}$ In addition, these ratings were highly correlated from pregnancy to postpartum, indicating stability over time.

Although the study sample was not demographically matched to the college students, preliminary results suggest blunted reactivity to emotional stimuli in nondepressed pregnant and postpartum women, indicating a difference in emotional processing during childbearing. This highlights the need to use appropriate stimuli sets and tasks for the measurement of these implicit processes (that vary depending on the research question) and to pay particular attention to the methodology and validity of the experimental design. Stimuli that have been validated with the appropriate sample (ie, pregnant and postpartum women) will provide important normative information with which future researchers can compare results of studies with this population. This enhances the scope and validity of tasks and experimental stimuli, similar to normative data used in neuropsychological tests that measure intelligence and memory.

\section{A specific model of affective reactivity}

One explanatory model of affective reactivity is guided by the Evaluative Space Model, ${ }^{72-75}$ which specifies that the 
affect system has evolved to automatically organize images into positive and negative valence categories. The positive and negative features merge into an implicit evaluation, influencing motivation to approach or avoid. ${ }^{72}$ In this model, the affect system is characterized by at least two asymmetries of response. First, the negativity bias is evidenced by larger responses (high negative valence ratings) to highly unpleasant than to equally extreme pleasant stimuli. This, along with other research, implies that all else being held constant, negative information has a stronger effect on behavior than positive information. ${ }^{76-78}$ The second asymmetry is the positivity offset, characterized by slightly positive responses to neutral stimuli. The default of the affect system when encountering neutral information is slightly positive, ${ }^{79}$ and because it is stable, it is regained quickly after unpleasant events. ${ }^{80}$

Negativity bias and positivity offset function separately, permitting flexible reactivity to stimuli of varied valence and intensity. For example, positivity offset invokes a stronger motivation (but not behavior) to explore than to withdraw when responding to neutral to mildly pleasant stimuli. Likewise, negativity bias invokes a stronger motivation to withdraw rather than explore when responding to equally valenced pleasant and unpleasant stimuli. Experimental tests of the Evaluative Space Model in healthy subjects support the model's conceptualization, facets, measurement, and temporal stability. ${ }^{81-83}$ Moreover, electrophysiological research has indicated that negativity bias is associated with a larger late positive potential $1^{77,84,85}$ and that positivity offset and negativity bias are differentially associated with two serotonin receptor genes. ${ }^{86}$

Looking at clinical populations, some data have shown that depressed women have significantly higher negativity bias and lower positivity offset than healthy controls, which indicates heightened implicit reactivity to negative information and decreased positivity to neutral-minimally positive information. Depressed postpartum women have also shown significantly lower valence and arousal ratings than healthy postpartum women, which suggests distorted implicit reactivity and a uniquely altered function of the affect system. ${ }^{63}$ These underlying, implicit affective processes may serve as potential endophenotypes that could be used to personalize treatment for women experiencing symptoms of depression in pregnancy or postpartum. In addition, they may serve as indicators of disrupted affect and allow for early or preventive intervention. However, additional support for this model in a variety of populations and clinical presentations, particularly in perinatal samples, as well the determination of biological substrates of these processes, is necessary.

\section{Multilevel measurement of attention processing and affective reactivity}

Although implicit biological and physiological processes associated with affective and cognitive processes have been defined in both healthy and clinical populations, very little research has examined these constructs across women in childbearing age. Psychophysiological data have helped to characterize substrates of affective reactivity and attentional processing to deepen our understanding of endophenotypes that span the continuum of health to psychopathology. In pregnancy and postpartum, they may help refine the role of neurobiological and physiological changes related specifically to emotional and cognitive functioning. For example, electrophysiological signals linked with active muscles are of central interest because of their value as indices, and possible mechanisms, of behavioral processes. Surface facial electromyography (EMG) includes measures of negative and positive facial expression, ${ }^{87}$ whereas startle blink modulation is a measure of the startle reflex to fearful or threatening information. Research using facial EMG has documented patterns of covert motor activity that differentiate both within and between emotional and cognitive processes ${ }^{88-90}$ and between healthy and clinical populations. ${ }^{53,91-93}$

Electrodermal activity includes measures of tonic levels of electrical conductance (skin conductance level) and changes as a function of task or in response to emotional stimuli (skin conductance response). Although facial EMG and startle modulation are sensitive to valence, electrodermal activity is a sensitive measure of arousal and has been shown to increase in response to emotional compared with neutral stimuli. ${ }^{94,95}$ In particular, research by Peter Lang and colleagues has examined the importance of these physiological indices in differentiating mood and anxiety disorders. ${ }^{48,52,53,95}$ Other measures are indices of specific processes (eg, negativity bias) that may manifest in multiple physiological systems (eg, patterns of facial EMG). The use of multiple measures is necessary to establish a thorough understanding of the underlying constructs and mechanisms of emotion and attention. In particular, it may provide important information regarding the physiological and biological components of emotional expression across pregnancy, allowing for a more nuanced classification and treatment of emotional disorders in pregnant and postpartum women. 
More specifically, identifying the neural substrates of affective reactivity and attentional processing may improve our understanding of how healthy individuals respond to emotional information, areas of the brain that show relative hyper- or hypoactivation as a result of clinical symptoms, and how these processes may be altered across childbearing. For example, depressive symptom reduction may be linked with increased activation of the anterior cingulate, an area that integrates affective with cognitive information to produce an adaptive goal-directed response. ${ }^{96,97}$ Moreover, women with major depressive disorder have shown increased activation of the amygdala to negative stimuli relative to healthy controls. ${ }^{96,98,99}$ In healthy women, the prefrontal cortex modulates or inhibits activity in the amygdala, thereby downregulating negative affect. ${ }^{100}$ This suggests the prefrontal cortex is a cognitive control mechanism governing perception of and attention to emotional stimuli. Further tests of these important brain areas in pregnant and postpartum women may help refine our understanding of the nature and course of affective responding during this transition.

Although neuroimaging has been examined in perinatal populations during passive viewing of emotional stimuli, the data are limited. Comparisons of attentional control tasks from pregnancy to postpartum or before and after treatment have not been studied. In one structural neuroimaging study, women between 2-4 weeks and 3-4 months postpartum were evaluated. An increase in gray matter volume, specifically in the prefrontal cortex, parietal lobes, and midbrain areas was observed. In addition, increased gray matter volume in the midbrain areas (ie, hypothalamus, substantia nigra, and amygdala) was associated with a mother's positive perception of her baby. ${ }^{101}$ In summary, the first months of motherhood in healthy women appear to be accompanied by structural changes associated with increased positive emotion toward infants. These adaptations may be altered by depressive or anxious symptoms or disorder. Future research should compare healthy pregnant and postpartum women with healthy women and should explore the effects of postpartum depression on these processes.

\section{Modifying attentional processing and affective reactivity may reduce depressive symptoms}

Behavioral tasks that aim to modify attentional processing may also function to reduce depression symptoms; ${ }^{102}$ however, the mechanisms that facilitate symptom improvement have yet to be fully investigated. In one study, attention training resulted in lower attention bias scores for negative stimuli among dysphoric individuals. ${ }^{103}$ The use of effortful cognitive strategies in recovery from depression, ${ }^{104}$ and the influence of attention bias manipulation on depressive symptoms in mild, moderate, and severe depression, have been investigated. ${ }^{105}$ Results of these studies reveal that modification of cognitive and attentional biases have direct effects on symptoms of depression, indicating them as potential targets for treatment. In addition, they are easy to deliver and tolerate, making them especially attractive as an adjunct to currently available treatments.

Few studies have examined reductions or modifications of these processes as a component of treatment in childbearing, despite its importance, ease of delivery, and potential to reduce negative effects on mother-infant interactions. Mothers have reliably shown rapid responses (within 800 milliseconds) toward infant signals, similar to the concept of an unconditioned response to a stimulus. ${ }^{106}$ This observation provides important information about how women respond to their infants, particularly indicating these responses occur outside of conscious awareness, and therefore could potentially be targeted in treatment via implicit modification tasks. Modifications of these processes may be especially important during pregnancy as opposed to postpartum because of the neurobiological and endocrine changes that occur. For example, neuroplasticity during pregnancy has been observed in preparation for motherhood, particularly in the brain regions of the hippocampus and amygdala. ${ }^{107-111}$ Notably, these changes persisted into late adulthood; however, these assumptions rely heavily on data from animal models. Future studies should examine whether these changes are similar in humans, as this may inform parental-infant interactions and may elucidate progression of these changes over time. Furthermore, modifications during pregnancy, as opposed to postpartum, minimize potential effect on the infant by correcting disruptions early on.

Established treatments for mood disorders have also been examined in perinatal samples, although their effect on implicit processing has not been widely studied. One study examined the effects of cognitive behavioral therapy compared with usual care on attentional processing in depressed pregnant women. ${ }^{112}$ Before treatment, depressed women showed diminished attentional bias for infant distress compared with a group of nondepressed pregnant women. ${ }^{37}$ However, after receiving cognitive behavioral therapy treatment (modified to focus on specific needs in pregnancy), women's attentional biases normalized and became comparable with pregnant women without depression. This indicates that treatment before childbirth can improve 
implicit attentional processing of infant stimuli, which may be directly related to positive infant outcomes. ${ }^{34}$ This observation supports the hypothesis that changes in explicit emotional responses to infants have positive outcomes for mother and baby and may potentially reflect alterations in underlying, implicit processes. Although existing treatments for depression in pregnancy and postpartum are efficacious, examining attentional processing and affectivity reactivity during treatment via modification tasks may optimize the therapeutic outcome for mother and baby.

\section{Identifying mechanisms of change is key to individualized treatment}

The next critical step in addressing this gap in knowledge is to investigate attentional processing and affective reactivity as potential mechanisms of action in treatment in pregnancy and postpartum. Future work could focus on this gap in the research by testing the hypothesis that a stronger affective reactivity to negative information (heightened negativity bias) motivates a pregnant woman to avoid the situation from which the negative information emerged. Treatment would focus on supporting the patient to habituate to the environment, which reduces the effects of the negative scenario over time. The patient would learn techniques to effectively manage her affective reactivity (similar to the concept of increasing distress tolerance) and adaptively respond to negative situations. In addition, having weaker affective reactivity to positive information may reveal a blunted reactivity to mildly pleasant images, which generates lower motivation to approach novel pleasant experiences. Treatment would focus on heightening affective reactivity to positive stimuli, thereby increasing motivation to approach and engage in pleasant situations.

With respect to attentional bias, researchers could test a model that posits that decreased attentional bias to infant distress leads postpartum women to respond maladaptively to their infants during times when they need comfort, potentially disrupt mother-infant interactions during early development, and through treatment, learn techniques to modify attentional biases, adaptively identify support systems in the environment to meet the infants' needs, and thus increase positive mother-infant interactions.

Finally, treatment may focus directly on helping mothers respond appropriately to their infant's cues. A substantial body of research has examined the importance of training mothers to be attentive and responsive to specific vocal and visual clues from their infant (ie, crying, facial expressions, other vocalizations) using models drawn from attachment theory. ${ }^{113-115}$ In depressed mothers, interventions targeting response to infant behaviors have shown improved mother-infant interactions. ${ }^{116,117}$ These results provide support for the beneficial effect of targeted maternal intervention to increase sensitivity and adaptive response to infant cues both in healthy and depressed mothers. The focus of attentional processing and affective reactivity to infant cues within these treatments may further enhance positive mother-infant interactions. This may be particularly important in dysregulated infants (ie, inconsolable, excessively crying, and unpredictable), given research showing that maternal behavior is less positive in irritable or colicky infants. ${ }^{118,119}$ We refer the reader to additional literature for specific studies on infant crying behavior and maternal interaction. ${ }^{120,121}$

\section{Conclusion}

Well-designed tests of attention processing and affective reactivity with validated stimuli sets of emotional images are needed to address a key barrier to understanding how emotion and attention serve as mechanisms of action in treatment for women in pregnancy and postpartum. Multiple biological, physiological, emotional, and cognitive changes occur during childbearing and affect the mother's view of herself, her baby, and the world in both adaptive and maladaptive ways. In particular, women become more attuned to their infants to provide adequate protection for themselves and their babies. In mothers with depressive symptoms during this critical time, attentional biases and affective reactivity are altered in a manner that is detrimental to their capacity to respond to environmental demands and infant distress.

Relevant to the mission of promoting women's mental health in pregnancy and in the postpartum period, the long-term goals of this work would be to characterize neurocognitive and affective features associated with healthy pregnancy and postpartum transitions. These normative data could be compared with data from women with common disorders such as depression and anxiety to adapt scalable training tasks to recalibrate attention and affect, evaluate the progressive changes in neurocognitive function during psychotherapy and psychopharmacotherapy, evaluate how attentional bias and affective reactivity function as causal mechanisms of remission and relapse, and use multilevel assessment of physiologic, affective, neurocognitive, and behavioral indices to characterize change in the perinatal phase. To achieve these goals, future research should examine the role of potential mechanisms of change in pregnancy and postpartum. 


\section{Acknowledgment}

Preparation of this article and the study was supported by funding from the Woman's Board of Northwestern Memorial Hospital and funding from the Francis Beidler Foundation.

\section{Disclosure}

The authors report no conflicts of interest in this work.

\section{References}

1. Jarrahi-Zadeh A, Kane FJ Jr, Van de Castlf RL, Lachenbruch PA, Ewing JA. Emotional and cognitive changes in pregnancy and early puerperium. Br J Psychiatry. 1969;115(524):797-805.

2. Kane FJ Jr, Harman WJ Jr, Keeler MH, Ewing JA. Emotional and cognitive disturbance in the early puerperium. $\mathrm{Br} J$ Psychiatry. 1968;114(506):99-102.

3. Pechtel P, Murray L, Brumariu LE, Lyons-Ruth K. Reactivity, regulation, and reward responses to infant cues among mothers with and without psychopathology: An fMRI review. Translational Dev Psychiatry. 2013;1(19673):1-17.

4. Anderson MV, Rutherford MD. Cognitive reorganization during pregnancy and the postpartum period: an evolutionary perspective. Evol Psychol. 2012;10(4):659-687.

5. Crawley RA, Dennison K, Carter C. Cognition in pregnancy and the first year post-partum. Psychol Psychother. 2003;76(Pt 1):69-84.

6. Pearson RM, Lightman SL, Evans J. Emotional sensitivity for motherhood: late pregnancy is associated with enhanced accuracy to encode emotional faces. Horm Behav. 2009;56(5):557-563.

7. Christensen H, Poyser C, Pollitt P, Cubis J. Pregnancy may confer a selective cognitive advantage. J Reprod Infant Psychol. 1999;17(1):7-25.

8. Russell JA, Douglas AJ, Ingram CD. Brain preparations for maternity adaptive changes in behavioral and neuroendocrine systems during pregnancy and lactation. An overview. Prog Brain Res. 2001;133:1-38.

9. Buckwalter JG, Buckwalter DK, Bluestein BW, Stanczyk FZ. Pregnancy and post partum: changes in cognition and mood. Prog Brain Res. 2001;133:303-319.

10. Buckwalter JG, Stanczyk FZ, McCleary CA, et al. Pregnancy, the postpartum, and steroid hormones: effects on cognition and mood. Psychoneuroendocrinology. 1999;24(1):69-84.

11. Wadhwa PD, Porto M, Garite TJ, Chicz-DeMet A, Sandman CA. Maternal corticotropin-releasing hormone levels in the early third trimester predict length of gestation in human pregnancy. Am J Obstet Gynecol. 1998;179(4):1079-1085.

12. Strathearn L, Fonagy P, Amico J, Montague PR. Adult attachment predicts maternal brain and oxytocin response to infant cues. Neuropsychopharmacology. 2009;34(13):2655-2666.

13. MacLeod C, Mathews A, Tata P. Attentional bias in emotional disorders. J Abnorm Psychol. 1986;95(1):15-20.

14. Pearson RM, Lightman SL, Evans J. Attentional processing of infant emotion during late pregnancy and mother-infant relations after birth. Arch Women Ment Health. 2011;14(1):23-31.

15. Posner MI, Snyder CR, Davidson BJ. Attention and the detection of signals. J Exp Psychol. 1980;109(2):160-174.

16. MacLeod C, Mathews A, Tata P. Attentional bias in emotional disorders. J Abnorm Psychol. 1986;95(1):15-20.

17. Ekman P, Friesen WV. Constants across cultures in the face and emotion. J Pers Soc Psychol. 1971;17(2):124-129.

18. Lang PJ, Bradley MM, Cuthbert BN. International affective picture system (IAPS): Affective ratings of pictures and instruction manual. Technical Report A-8. Gainesville, FL: University of Florida; 2008.

19. Joormann J, Gotlib IH. Emotion regulation in depression: relation to cognitive inhibition. Cogn Emotion. 2010;24(2):281-298.

20. Gotlib IH, Krasnoperova E, Yue DN, Joormann J. Attentional biases for negative interpersonal stimuli in clinical depression. JAbnorm Psychol. 2004;113(1):121-135.
21. Ohman A, Mineka S. Fears, phobias, and preparedness: toward an evolved module of fear and fear learning. Psychol Rev. 2001;108(3):483-522.

22. Georgiou GA, Bleakley C, Hayward J, et al. Focusing on fear: Attentional disengagement from emotional faces. Vis Cogn. 2005;12(1):145-158.

23. Brosch T, Sander D, Scherer KR. That baby caught my eye ... attention capture by infant faces. Emotion. 2007;7(3):685-689.

24. Peckham AD, McHugh RK, Otto MW. A meta-analysis of the magnitude of biased attention in depression. Depress Anxiety. 2010;27(12):1135-1142.

25. Mogg K, Bradley BP, Williams R. Attentional bias in anxiety and depression: the role of awareness. Br J Clin Psychol. 1995;34(Pt 1): $17-36$.

26. Joormann J, Nee DE, Berman MG, Jonides J, Gotlib IH. Interference resolution in major depression. Cogn Affect Behav Neurosci. 2010;10(1): 21-33.

27. McCabe SB, Gotlib IH. Selective attention and clinical depression: performance on a deployment-of-attention task. J Abnorm Psychol. 1995;104(1):241-245

28. Sanchez A, Vazquez C, Marker C, LeMoult J, Joormann J. Attentional disengagement predicts stress recovery in depression: an eye-tracking study. J Abnorm Psychol. 2013;122(2):303-313.

29. Beck AT, Emergy G, Greenberg RL. Anxiety Disorders and Phobias: A Cognitive Perspective. New York, NY: Basic Books; 1985.

30. Mogg K, Bradley BP. A cognitive-motivational analysis of anxiety. Behav Res Ther. 1998;36(9):809-848.

31. Mogg K, Garner M, Bradley BP. Anxiety and orienting of gaze to angry and fearful faces. Biol Psychol. 2007;76(3):163-169.

32. MacLeod C, Rutherford E, Campbell L, Ebsworthy G, Holker L. Selective attention and emotional vulnerability: assessing the causal basis of their association through the experimental manipulation of attentional bias. J Abnorm Psychol. 2002;111(1):107-123.

33. Stark MA. Directed attention in normal and high-risk pregnancy J Obstet Gynecol Neonatal Nurs. 2006;35(2):241-249.

34. Landry SH, Smith KE, Swank PR. Responsive parenting: establishing early foundations for social, communication, and independent problemsolving skills. Dev Psychol. 2006;42(4):627-642.

35. De Wolff MS, van Ijzendoorn MH. Sensitivity and attachment: a metaanalysis on parental antecedents of infant attachment. Child Dev. 1997;68(4):571-591.

36. Thompson-Booth C, Viding E, Mayes LC, Rutherford HJ, Hodsoll S, McCrory EJ. Here's looking at you, kid: attention to infant emotional faces in mothers and non-mothers. Dev Sci. 2014;17(1):35-46.

37. Pearson RM, Cooper RM, Penton-Voak IS, Lightman SL, Evans J. Depressive symptoms in early pregnancy disrupt attentional processing of infant emotion. Psychol Med. 2010;40(4):621-631.

38. Siddiqui A, Hägglöf B. Does maternal prenatal attachment predict postnatal mother-infant interaction? Early Hum Dev. 2000;59(1):13-25.

39. Shin H, Park YJ, Kim MJ. Predictors of maternal sensitivity during the early postpartum period. $J A d v$ Nurs. 2006;55(4):425-434.

40. Hellgren C, Bannbers E, Åkerud H, Risbrough V, Poromaa IS. Decreased startle modulation during anticipation in the postpartum period in comparison to late pregnancy. Arch Women Ment Health. 2012;15(2):87-94.

41. Bylsma LM, Morris BH, Rottenberg J. A meta-analysis of emotional reactivity in major depressive disorder. Clin Psychol Rev. 2008;28(4):676-691.

42. Swain JE, Leckman JF, Mayes LC, Feldman R, Constable RT, Schultz RT. Neural substrates and psychology of human parent-infant attachment in the postpartum. Biol Psychiatry. 2004;55(8):153S.

43. Strathearn L, Li J, Fonagy P, Montague PR. What's in a smile? Maternal brain responses to infant facial cues. Pediatrics. 2008;122(1):40-51.

44. Swain JE, Lorberbaum JP, Kose S, Strathearn L. Brain basis of early parent-infant interactions: psychology, physiology, and in vivo functional neuroimaging studies. $J$ Child Psychol Psychiatry. 2007;48(3-4):262-287. 
45. Bartels A, Zeki S. The neural correlates of maternal and romantic love. Neuroimage. 2004;21(3):1155-1166.

46. Nitschke JB, Nelson EE, Rusch BD, Fox AS, Oakes TR, Davidson RJ. Orbitofrontal cortex tracks positive mood in mothers viewing pictures of their newborn infants. Neuroimage. 2004;21(2):583-592.

47. Kim P, Leckman JF, Mayes LC, Newman MA, Feldman R, Swain JE. Perceived quality of maternal care in childhood and structure and function of mothers' brain. Dev Sci. 2010;13(4):662-673.

48. Lang PJ, Greenwald MK, Bradley MM, Hamm AO. Looking at pictures: affective, facial, visceral, and behavioral reactions. Psychophysiology. 1993;30(3):261-273.

49. Yik MSM, Russell JA, Barrett LF. Structure of self-reported current affect: Integration and beyond. J Pers Soc Psychol. 1999;77(3): 600-619.

50. Lee TM, Ng EH, Tang SW, Chan CC. Effects of sad mood on facial emotion recognition in Chinese people. Psychiatry Res. 2008; 159(1-2):37-43.

51. Joormann J, Gotlib IH. Is this happiness I see? Biases in the identification of emotional facial expressions in depression and social phobia. J Abnorm Psychol. 2006;115(4):705-714.

52. Cuthbert BN, Lang PJ, Strauss C, Drobes D, Patrick CJ, Bradley MM. The psychophysiology of anxiety disorder: fear memory imagery. Psychophysiology. 2003;40(3):407-422.

53. Lang PJ, McTeague LM. The anxiety disorder spectrum: fear imagery, physiological reactivity, and differential diagnosis.Anxiety Stress Coping. 2009;22(1):5-25.

54. Ingram RE, Bernet $\mathrm{CZ}$, McLaughlin SC. Attentional allocation processes in individuals at risk for depression. Cognit Ther Res. 1994;18(4):317-332.

55. Ingram RE, Ritter J. Vulnerability to depression: cognitive reactivity and parental bonding in high-risk individuals. $J$ Abnorm Psychol. 2000;109(4):588-596.

56. Tronick E, Beeghly M. Infants' meaning-making and the development of mental health problems. Am Psychol. 2011;66(2):107-119.

57. Grace SL, Evindar A, Stewart DE. The effect of postpartum depression on child cognitive development and behavior: a review and critical analysis of the literature. Arch Women Ment Health. 2003;6(4):263-274.

58. Joormann J, Talbot L, Gotlib IH. Biased processing of emotional information in girls at risk for depression. J Abnorm Psychol. 2007;116(1):135-143.

59. Gil $\mathrm{S}$, Teissèdre $\mathrm{F}$, Chambres $\mathrm{P}$, Droit-Volet $\mathrm{S}$. The evaluation of emotional facial expressions in early postpartum depression mood: a difference between adult and baby faces? Psychiatry Res. 2011;186(2-3):281-286.

60. Stein A, Arteche A, Lehtonen A, et al. Interpretation of infant facial expression in the context of maternal postnatal depression. Infant Behav Dev. 2010;33(3):273-278.

61. Flanagan TJ, White H, Carter BG. Differential impairments in emotion face recognition in postpartum and nonpostpartum depressed women. J Affect Disord. 2011;128(3):314-318.

62. Arteche A, Joormann J, Harvey A, et al. The effects of postnatal maternal depression and anxiety on the processing of infant faces. $J$ Affect Disord. 2011;133(1-2):197-203.

63. Gollan JK, Hoxha D, Getch S, Sankin L, Michon R. Affective information processing in pregnancy and postpartum with and without major depression. Psychiatry Res. 2013;206(2-3):206-212.

64. Roos A, Robertson F, Lochner C, Vythilingum B, Stein DJ. Altered prefrontal cortical function during processing of fear-relevant stimuli in pregnancy. Behav Brain Res. 2011;222(1):200-205.

65. Kinsley CH, Lambert KG. The maternal brain. Sci Am. 2006;294(1):72-79.

66. Seifritz E, Esposito F, Neuhoff JG, et al. Differential sex-independent amygdala response to infant crying and laughing in parents versus nonparents. Biol Psychiatry. 2003;54(12):1367-1375.

67. Leibenluft E, Gobbini MI, Harrison T, Haxby JV. Mothers' neural activation in response to pictures of their children and other children. Biol Psychiatry. 2004;56(4):225-232.
68. Silverman ME, Loudon H, Safier M, et al. Neural dysfunction in postpartum depression: an fMRI pilot study. CNS Spectr. 2007;12(11):853-862.

69. Moses-Kolko EL, Perlman SB, Wisner KL, James J, Saul AT, Phillips ML. Abnormally reduced dorsomedial prefrontal cortical activity and effective connectivity with amygdala in response to negative emotional faces in postpartum depression. Am J Psychiatry. 2010;167(11):1373-1380

70. Stein A, Murphy S, Arteche A, et al. Effects of reboxetine and citalopram on appraisal of infant facial expressions and attentional biases. J Psychopharmacol. 2012;26(5):670-676.

71. Rosebrock LE, Sankin L, Hoxha D, Gollan JK. Affective reactivity in pregnancy and postpartum: Current tasks and future directions. Perinatal Mental Health Meeting; November 6-8; 2013; Chicago, IL.

72. Cacioppo JT, Berntson GG, Larsen JT, Poehlmann KM, Ito TA. The psychophysiology of emotion. In: Lewis M, Haviland JM, editors. Handbook of Emotions. Vol 2. New York, NY: Guilford Press; 2000:173-191.

73. Cacioppo JT, Berntson GG. Relationship between attitudes and evaluative space: A critical review, with emphasis on the separability of positive and negative substrates. Psychol Bull. 1994;115(3):401-423.

74. Cacioppo JT, Gardner WL, Berntson GG. Beyond bipolar conceptualizations and measures: the case of attitudes and evaluative space. Pers Soc Psychol Rev. 1997;1(1):3-25.

75. Cacioppo JT, Gardner WL, Berntson GG. The affect system has parallel and integrative processing components: Form follows function. $J$ Soc Psychol. 1999;76(5):839-855.

76. Baumeister RF, Bratslavsky E, Finkenauer C, Vohs KD. Bad is stronger than good. Rev Gen Psychol. 2001;5(4):323-370.

77. Ito TA, Larsen JT, Smith NK, Cacioppo JT. Negative information weighs more heavily on the brain: the negativity bias in evaluative categorizations. J Pers Soc Psychol. 1998;75(4):887-900.

78. Kahneman D, Tversky A. Choices, values, and frames. Am Psychol. 1984;39(4):341-350

79. Diener E, Seligman ME. Very happy people. Psychol Sci. 2002;13(1):81-84.

80. Gilbert DT, Pinel EC, Wilson TD, Blumberg SJ, Wheatley TP. Immune neglect: a source of durability bias in affective forecasting. J Pers Soc Psychol. 1998;75(3):617-638.

81. Larsen JT, Norris CJ, McGraw AP, Hawkley LC, Cacioppo JT. The evaluative space grid: A single-item measure of positivity and negativity. Cogn Emotion. 2009;23(3):453-480.

82. Ito TA, Cacioppo JT. Variations on a human universal: Individual differences in positivity offset and negativity bias. Cogn Emotion. 2005;19(1):1-26.

83. Norris CJ, Larsen JT, Crawford LE, Cacioppo JT. Better (or worse) for some than others: Individual differences in the positivity offset and negativity bias. $J$ Res Pers. 2011;45(1):100-111.

84. Ito TA, Cacioppo JT. Electrophysiological evidence of implicit and explicit categorization processes. J Exp Soc Psychol. 2000;36(6):660-676.

85. Smith NK, Larsen JT, Chartrand TL, Cacioppo JT, Katafiasz HA, Moran KE. Being bad isn't always good: affective context moderates the attention bias toward negative information. J Pers Soc Psychol. 2006;90(2):210-220.

86. Ashare RL, Norris CJ, Wileyto EP, Cacioppo JT, Strasser AA. Individual differences in positivity offset and negativity bias: Gender-specific associations with two serotonin receptor genes. Pers Individ Dif. 2013;55(5):469-473.

87. Tassinary LG, Cacioppo JT, Vanman EJ. The skeletomotor system: surface electromyography. In: Cacioppo JT, Tassinary LG, Berntson GG, editors. Handbook of Psychophysiology. 3rd ed. New York: Cambridge University Press; 2007:267-299.

88. Cacioppo JT, Klein DJ, Berntson GG, Hatfield E. The psychophysiology of emotion. In: Lewis M, Haviland JM, editors. Handbook of Emotions. New York, NY: Guilford Press; 1993:119-142. 
89. Tassinary LG, Cacioppo JT. Unobservable facial actions and emotion. Psychol Sci. 1992;3(1):28-33.

90. Fridlund AJ, Izard CE. Electromyographic studies of facial expressions of emotions and patterns of emotion. In: Cacioppo JT, Petty RE, editors. Social Psychophysiology: A Sourcebook. New York, NY: Guilford Press; 1983:243-286.

91. Hazlett RL, McLeod DR, Hoehn-Saric R. Muscle tension in generalized anxiety disorder: elevated muscle tonus or agitated movement? Psychophysiology. 1994;31(2):189-195.

92. Orr SP, Pitman RK, Lasko NB, Herz LR. Psychophysiological assessment of posttraumatic stress disorder imagery in World War II and Korean combat veterans. J Abnorm Psychol. 1993;102(1):152-159.

93. van Boxtel A, Goudswaard P, Janssen K. Absolute and proportional resting EMG levels in muscle contraction and migraine headache patients. Headache. 1983;23(5):215-222.

94. Mardaga S, Hansenne M. Autonomic aspect of emotional response in depressed patients: relationships with personality. Neurophysiol Clin. 2009;39(4-5):209-216.

95. Cuthbert BN, Schupp HT, Bradley MM, Birbaumer N, Lang PJ. Brain potentials in affective picture processing: covariation with autonomic arousal and affective report. Biol Psychol. 2000;52(2):95-111.

96. Mayberg HS, Liotti M, Brannan SK, et al. Reciprocal limbic-cortical function and negative mood: converging PET findings in depression and normal sadness. Am J Psychiatry. 1999;156(5):675-682.

97. Pizzagalli D, Pascual-Marqui RD, Nitschke JB, et al. Anterior cingulate activity as a predictor of degree of treatment response in major depression: evidence from brain electrical tomography analysis. $\mathrm{Am}$ J Psychiatry. 2001;158(3):405-415.

98. Dannlowski U, Ohrmann P, Bauer J, et al. Amygdala reactivity predicts automatic negative evaluations for facial emotions.Psychiatry Res. 2007;154(1):13-20.

99. Siegle GJ, Thompson W, Carter CS, Steinhauer SR, Thase ME. Increased amygdala and decreased dorsolateral prefrontal BOLD responses in unipolar depression: related and independent features. Biol Psychiatry. 2007;61(2):198-209.

100. Jackson DC, Mueller CJ, Dolski I, et al. Now you feel it, now you don't: frontal brain electrical asymmetry and individual differences in emotion regulation. Psychol Sci. 2003;14(6):612-617.

101. Kim P, Leckman JF, Mayes LC, Feldman R, Wang X, Swain JE. The plasticity of human maternal brain: longitudinal changes in brain anatomy during the early postpartum period. Behav Neurosci. 2010;124(5):695-700.

102. Bowler JO, Mackintosh B, Dunn BD, Mathews A, Dalgleish T, Hoppitt L. A comparison of cognitive bias modification for interpretation and computerized cognitive behavior therapy: effects on anxiety, depression, attentional control, and interpretive bias. J Consult Clin Psychol. 2012;80(6):1021-1033.

103. Wells TT, Beevers CG. Biased attention and dysphoria: Manipulating selective attention reduces subsequent depressive symptoms. Cogn Emotion. 2010;24(4):719-728.

104. Watkins ER, Moulds M. Revealing negative thinking in recovered major depression: a preliminary investigation. Behav Res Ther. 2007;45(12):3069-3076.

105. Baert S, De Raedt R, Schacht R, Koster EH. Attentional bias training in depression: therapeutic effects depend on depression severity. J Behav Ther Exp Psychiatry. 2010;41(3):265-274.
106. Papousek H, Papousek M. Biological basis of social interactions: implications of research for an understanding of behavioural deviance. J Child Psychol Psychiatry. 1983;24(1):117-129.

107. Pawluski JL, Galea LA. Hippocampal morphology is differentially affected by reproductive experience in the mother. $J$ Neurobiol. 2006;66(1):71-81

108. Rasia-Filho AA, Fabian C, Rigoti KM, Achaval M. Influence of sex, estrous cycle and motherhood on dendritic spine density in the rat medial amygdala revealed by the Golgi method. Neuroscience. 2004;126(4):839-847.

109. Kinsley CH, Lambert KG. Reproduction-induced neuroplasticity: natural behavioural and neuronal alterations associated with the production and care of offspring. J Neuroendocrinol. 2008;20(4):515-525.

110. Kinsley CH, Trainer R, Stafisso-Sandoz G, et al. Motherhood and the hormones of pregnancy modify concentrations of hippocampal neuronal dendritic spines. Horm Behav. 2006;49(2):131-142.

111. Lambert KG, Love G, Stevens L, Klein SL, Conway AF, Kinsley CH. Nissl analyses indicate maternal-induced hippocampal and amygdalar neuronal/glia plasticity in wild-caught Norway rats. Annual Meeting of the Society for Neuroscience; 2005; Washington, DC.

112. Pearson RM, O'Mahen H, Burns A, et al. The normalisation of disrupted attentional processing of infant distress in depressed pregnant women following Cognitive Behavioural Therapy. J Affect Disord. 2013;145(2):208-213.

113. Whitt JK, Casey PH. The mother-infant relationship and infant development: the effect of pediatric intervention. Child Dev. 1982;53(4):948-956.

114. Erickson MF, Korfmacher J, Egeland BR. Attachments past and present: Implications for therapeutic intervention with mother-infant dyads. Dev Psychopathol. 1992;4(4):495-507.

115. van den Boom DC. The influence of temperament and mothering on attachment and exploration: an experimental manipulation of sensitive responsiveness among lower-class mothers with irritable infants. Child Dev. 1994;65(5):1457-1477.

116. Jung V, Short R, Letourneau N, Andrews D. Interventions with depressed mothers and their infants: modifying interactive behaviours. J Affect Disord. 2007;98(3):199-205.

117. Clark R, Tluczek A, Wenzel A. Psychotherapy for postpartum depression: a preliminary report. Am J Orthopsychiatry. 2003;73(4):441-454.

118. van den Boom DC, Hoeksma JB. The effect of infant irritability on mother-infant interaction: A growth-curve analysis. Dev Psychol. 1994;30(4):581-590.

119. Räihä H, Lehtonen L, Huhtala V, Saleva K, Korvenranta H. Excessively crying infant in the family: mother-infant, father-infant and motherfather interaction. Child Care Health Dev. 2002;28(5):419-429.

120. Barr RG. Excessive crying. In: Sameroff AJ, Lewis M, Miller SM, editors. Handbook of Developmental Psychopathology. New York: Springer; 2000:327-350

121. James-Roberts IS, Conroy S, Wilsher K. Bases for maternal perceptions of infant crying and colic behaviour. Arch Dis Child. 1996;75(5):375-384.

Neuroscience and Neuroeconomics

\section{Publish your work in this journal}

Neuroscience and Neuroeconomics is an international, peer-reviewed, open access journal focusing on the identification of brain structures and measurement of neural activity related to behavior, behavioral predictions, and decision making in health and disease. The manuscrip management system is completely online and includes a very quick and

\section{Dovepress}

fair peer-review system. Visit http://www.dovepress.com/testimonials. 